\title{
Nanotechnology: A Spark to the Use of Plant Origin Bioactive Compounds in Therapeutics
}

\section{Sudesh Kumar Yadav*}

Plant Metabolic Engineering and Nanobiology, Biotechnology Division, CSIR-Institute of Himalayan Bioresource Technology, India

*Corresponding author: Sudesh Kumar Yadav, Plant Metabolic Engineering and Nanobiology, Biotechnology Division, CSIR-Institute of Himalayan Bioresource Technology, Palampur-176061, India, Tel: 01894-233339; E-mail: sudeshkumar@ihbt.res.in

Rec date: Jan 22, 2015; Acc date: Feb 20, 2015; Pub date: Feb 24, 2015

Copyright: () 2015 Yadav SK. This is an open-access article distributed under the terms of the Creative Commons Attribution License, which permits unrestricted use, distribution, and reproduction in any medium, provided the original author and source are credited.

\section{Short Communication}

With the advent of LC-MS, identification and characterization of compounds from plant origin has become very easy now a day. In this effort, several plant-based compounds have been identified possessing therapeutic propensities. This category includes cruciferous vegetables, garlic, ginger, onions, soybeans, and turmeric [1]. Such efforts propel us for large-scale screening of taxonomically authenticated dietary, edible and medicinally important plants for isolation of many more bioactive compounds. Unfortunately, limitation to the use of such compounds in clinical remedies is that of their bioavailability and targeted delivery [2]. Now nanotechnology seems to have potential solutions to both of these limitations. Encapsulation of bioactive compounds using biodegradable matrices like chitosan, polylactides etc. can enhance the solubility, controlled release as well as provide surface to the ligands for targeted delivery [3]. Hence, there is a need to provide the spark to bioactive compounds using nanotechnology for their effective use in therapeutics.

\section{Plants and bioactive compounds}

A variety of plant species have been explored for the bioactive compounds of enormous use. Some of the bioactive compounds like quercetin, quercitrin, podophyllotoxin, catechin, epicatechin, betulin, steviosides, and curcumin have been pharmacologically found useful for human health. Quercetin, an abundant flavonoid, present in plants like berries, apples, radish, broccoli, sea buckthorn is well known for its antioxidant activity [4]. While quercitrin holds an important place in ameliorating the reactive oxygen species induced oxidative stress in the body. Quercitrin is rich in plants like Albizia chinensis, onions, green tea, apples and Ginkgo biloba. It also possessed activities like anti-malarial, anti-inflammatory and inhibition of acetylcholinesterase [5]. Stevioside, a nontoxic natural non-caloric sweetener is very useful for diabetics. It is mainly extracted from the leaves of Stevia rebaudiana (Bertoni) and is considered to be a good anti-diabetic drug as it regulates the concentration of glucose in blood by secreting more insulin [6]. Podophyllotoxin is an anticancer molecule and found in larger concentrations in the rhizome of American Mayapple [7]. Catechin and epicatechin polyphenols are present in most abundance in tea. They possess neuroprotective properties and thereby prevent the neuronal cell death. These compounds of tea also reported for antitumorogenic, anti-oxidative, anti-mutagenic, and anti-pathogenic. Hence, catechins and epicatechins are being widely used an important constituent in anti-aging beauty products, food and nutraceuticals [8]. Instead of having innumerable astonishing properties of interest to mankind, these bioactive molecules render poor permeability, instability and extensive first pass metabolism before reaching the systemic circulation, deprived intestinal absorption, less bioavailability, less persistence, and degradation in metabolism.

\section{Nanoencapsulation: biodegradable polymers and methods}

In view of the advanced properties possessed by biomolecules, efforts have been put forward to overcome their limitations using nanotechnological aspects. These compounds have been encapsulated into a wide array of biodegradable polymer nanoparticles (NPs) to enhance their efficacy, stability, and utility. Various polymers have been employed for the encapsulation of biomolecules into a nanoformulation by using different methodologies. Use of biodegradable polymers offers an advantage being non-toxic and easily metabolised by our body system. Commonly used polymers are polylactic acid (PLA), poly-D,L-lactide-co-glycolide (PLGA), poly caprolactone (PCL), and chitosan. PLA is degraded to lactic acid in the body during carbohydrate metabolism. PLA has been used for the encapsulation of bioactive molecules like quercetin, savoxepine, ordinonin and taxol. PLGA is metabolized into lactic acid and glycolic acid that rendered minimum toxicity in the body. Paclitaxel, xanthone, insulin have been encapsulated into PLGA polymer. PCL is easily degraded in the body by hydrolysis of the ester linkages present in it. PCL polymer has been used for the encapsulation of taxol, and insulin. Gelatin holds a good place in food and medical products and is used for nanoencapsulation because it renders no toxicity. It is a polyampholyte containing both cationic and anionic groups along with hydrophilic group that serves as a useful polymer for nanoencapsulation. Choloroquine, paclitaxel, and bovine serum albumin has been successfully encapsulated into gelatine polymer [3].

Commonly used methods for the synthesis of NPs are solvent evaporation method, salting out, nanoprecipitation, and dialysis. In solvent evaporation method, both the polymer and drug molecules are dissolved in volatile organic solvent and are sonicated [9]. The salting out method is based upon the principle of separation of water miscible solvents from the aqueous solution by adding salts like magnesium chloride and calcium chloride. It offers an advantage to minimize the stress to any of the bioactive molecule being encapsulated [10]. In nanoprecipitation method, the drug and polymers are dissolved in an organic solvent like dichloromethane, acetone and then added dropwise to the mixture of water/methanol solution which decreases the interfacial tension. The solvent diffuses out and the bioactive and polymer precipitates in the form of NPs [11]. Dialysis method is based on the principle of change in osmotic pressure. The bioactive, polymer and surfactants are placed in a dialysis bag and kept in a solvent. The passive transport of solvent through the membrane leads to the formation of homogeneous suspension of NPs [12].

\section{Improved characteristics of bioactive nanoencapsulates}

Nanotechnology has significantly improved the performance of many bioactive molecules in terms of efficacy, safety and patient compliance. It manifests the usage of a variety of materials. It 
Citation: Yadav SK (2015) Nanotechnology: A Spark to the Use of Plant Origin Bioactive Compounds in Therapeutics. Single Cell Biol 4: 108. doi:10.4172/2168-9431.1000108

Page 2 of 2

facilitates smart designing and surface modifications in order to tag large number of molecular, biological and chemical entities. The modified surface properties aid to increase the solubility and biocompatibility of bioactive molecules [13]. In vivo studies have suggested the improved solubility, stability, bioavailability of bioactives upon encapsulation using polymers like polyvinylpyrrolidone, polyethylene glycol, PLA, PLGA [14]. The protection and controlled release of bioactive compounds at the right time and the right place can be made feasible by nanoencapsulation. The barricade faced by the natural product formulation is their structural instability in biological systems and untimely drug loss during the metabolism. The low solubility of bioactives in aqueous soluble can act improved by encapsulating them into soluble polymers [15]. The aqueous solubility of quercetin has been greatly enhanced by encapsulating into PLA polymer [4]. Stevioside has a problem of low intestinal absorption that can be solved by encapsulating into PLA polymer and improved its bioavailability and retention in the body [6]. The nanotechnology also aims at better targeting of the molecules at the desired site. The ligand coupled nanosystems have been developed for the targeted delivery. It enhances the cellular uptake and the retention time via receptor mediated endocytosis. The nanoencapsulates can cross the endothelial and epithelial barriers by transcytosis [14]. Different methods of nanoencapsulation are used for development of targeted delivery systems to minimize and delay the negative effects.

\section{References}

1. Mehta RG, Murillo G, Naithani R, Peng X (2010) Cancer chemoprevention by natural products: how far have we come? Pharm Res 27: 950-961.

2. Kumari A, Yadav SK (2011) Cellular interactions of therapeutically delivered nanoparticles. Expert Opin Drug Deliv 8: 141-151.

3. Kumari A, Yadav SK, Yadav SC (2010) Biodegradable polymeric nanoparticles based drug delivery systems. Colloids Surf B Biointerfaces 75: $1-18$.
4. Kumari A, Yadav SK, Pakade YB, Singh B, Yadav SC (2010) Development of biodegradable nanoparticles for delivery of quercetin. Colloids Surf B Biointerfaces 80: 184-192.

5. Kumari A, Yadav SK, Pakade YB, Kumar V, Singh B, et al. (2011) Nanoencapsulation and characterization of Albizia chinensis isolated antioxidant quercitrin on PLA nanoparticles. Colloids Surf B Biointerfaces 82: 224-232.

6. Barwal I, Sood A, Sharma M, Singh B, Yadav SC (2013) Development of stevioside Pluronic-F-68 copolymer based PLA-nanoparticles as an antidiabetic nanomedicine. Colloids Surf B Biointerfaces 101: 510-516.

7. Yadav R, Kumar D, Kumari A, Yadav SK (2014) Encapsulation of podophyllotoxin and etoposide in biodegradable poly-d,l-lactide nanoparticles improved their anticancer activitivity Journal of Microencapsulation 31: 211-219.

8. Yadav R, Kumar D, Kumari A, Yadav SK (2014) Encapsulation of catechin and epicatechin on bsa nps improved their stability and antioxidant Potential. excli Journal 13: 331-346.

9. Raghuvanshi RS, Katare YK, Lalwani K, Ali MM, Singh O, et al. (2002) Improved immune response from biodegradable polymer particles entrapping tetanus toxoid by use of different immunization protocol and adjuvants. Int J Pharm 245: 109-121.

10. Pinto Reis C, Neufeld RJ, Ribeiro AJ, Veiga F (2006) Nanoencapsulation I. Methods for preparation of drug-loaded polymeric nanoparticles. Nanomedicine 2: 8-21.

11. Barichello JM, Morishita M, Takayama K, Nagai T (1999) Encapsulation of hydrophilic and lipophilic drugs in PLGA nanoparticles by the nanoprecipitation method. Drug Dev Ind Pharm 25: 471-476.

12. Nagavarma BVN, Yadav HKS, Ayaz A, Vasudha LS, Kumar SHG (2012) Different Techniques for Preparation of Polymeric Nanoparticles - A Review. Asian Journal of Pharmaceutical and Clinical Research 5: 16-23.

13. Baimarck Y (2009) Journal of Applied Science, 12: 263-270.

14. Kumari A, Kumar V, Yadav SK (2012) Nanotechnology: A Tool to Enhance Therapeutic Values of Natural Plant Products Trends Med Res 7: 34-42.

15. McNeil SE (2005) Nanotechnology for the biologist. J Leukoc Biol 78: 585-594. 